\title{
EVALUATION OF “CALCULATING" HELPERS BASED ON THIRD-PARTY OBSERVATION IN ADULTS AND CHILDREN
}

\author{
Reiki KISHIMOTO' $^{1)}$, Shoji ITAKURA ${ }^{1)}$, Kazuo FUJITA $^{1)}$, and Kazuhide HASHIYA ${ }^{2)}$ \\ ${ }^{1)}$ Kyoto University, Japan \\ ${ }^{2)}$ Kyushu University, Japan
}

\begin{abstract}
In the context of reciprocity, behaving cooperatively only when it enhances one's reputation is a strategy that brings reputational benefits at minimal cost; however, if other members of society notice an individual employing such a strategy, any accumulated positive reputation may be negated. The present study addresses the development of this social judgement by examining how preschoolers and adults evaluate agents with or without reputational management. We presented Publiconly Helpers (cooperative only when they were seen) with Private-only Helpers (cooperative only when they were not seen) or Unconditional Non-Helpers (never cooperative regardless of being seen). Results showed that children less preferred Public-only Helpers presented with Private-only Helpers than with Unconditional Non-Helpers. Adults avoided Public-only Helpers irrespective of compared agents. Our findings indicate that although preschoolers' evaluations of reputational management are not as salient as those of adults, they less prefer people who provide help to gain personal reputational benefits.
\end{abstract}

Key words: calculating/strategic agent, third-party evaluation, indirect reciprocity, reputational management

\section{INTRODUCTION}

In natural and social science fields that address the origins of sociality, cooperation has become a central issue (Fehr, 2004; Nowak, 2006). Cooperation is widely observed in various species (Bshary \& Grutter, 2006; Trivers, 1971); however, in many species,

\footnotetext{
Reiki Kishimoto is currently affiliated with Department of Psychology, Aichi University, Japan. Shoji Itakura is currently affiliated with Doshisha University for Baby Science, Doshisha University, Japan.

We deeply appreciate the children and their mothers, and everyone for participating in the study, and Anderson J. R. for his insightful comments on the manuscript. We also thank H. Chijiiwa, K. Urata, K. Maeyama, M. Arahori, M. Ueno, N. Matsushima, R. Mizobata, S. Iwasaki, S. Takagi, Y. Nagae, Y. Uto, and Y. Akiyoshi for their help in conducting this study, E. Ogura for her help in recruiting participants and A. Kimura, D. Yokohata and Y. Ojiro for their contributions to the data analysis.

This study was financially supported by Grant-in-Aid for Scientific Research on Innovative Areas Grant Number JP25118003 “The Evolutionary Origin and Neural Basis of the Empathetic Systems" and Grant Number JP18H04200 "Construction of the Face-Body Studies in Transcultural Conditions" and by the JSPS Grant-in-Aide for Scientific Research, Grant Numbers (B) JP19H04431, (C) JP18K02461, (B) JP17KT0057 (C) JP17KT0139, and (B) JP26280049 to K.H., (S) Grant Number JP16H06301 to K.F. and (A) Grant Number JP25245067 to S.I. and Grant-in-Aid for JSPS Fellows Grant Number JP17J07232 and Grant-inAid for Research Activity Start-up Grant Number JP19K23391 to R.K.

Correspondence concerning this article should be addressed to Reiki Kishimoto, Department of Psychology, Aichi University, Japan (e-mail: kishimoto.r.k@gmail.com).
} 
individuals are not indiscriminate cooperators. In fact, field and laboratory studies of social species, including nonhuman primates and non-mammals (e.g., de Waal, 1989; Milinski, 1987; Wilkinson, 1984), have identified selective reciprocity with partners, generally based on expectations of receiving helping or altruistic behavior in return.

This selectiveness also applies to our own social species. With a set of speciesunique skills and motivations for cooperation (Tomasello, 2009), human adults are often selectively prosocial with others who have previously provided benefits (Trivers, 1971; McCabe, Houser, Ryan, Smith, \& Trouard, 2001). However, in early stages of development, humans tend to be less discriminating (Tomasello, 2009), with studies showing that, even when no reward is expected, infants will help others by providing information (Liszkowski, Carpenter, Striano, \& Tomasello, 2006; Liszkowski, Carpenter, \& Tomasello, 2008).

However, in ontogeny—-between toddlerhood and preschool—children's relatively indiscriminate cooperativeness becomes modulated by their expectations of reciprocity (Tomasello, 2009). Observations of nursery-school pupils and laboratory studies have shown that prosocial behavior becomes selective at this point, based on the familiarity between individuals or on having received prior prosocial favors from an individual (Fujisawa, Kutsukake, \& Hasegawa, 2008; Olson \& Spelke, 2008). From a theoretical perspective, there is debate concerning whether the psychological background of discriminative cooperation is based on reciprocal altruism or direct reciprocity (Tomasello, 2009).

Human adults tend to cooperate with individuals who are cooperative with other individuals. This type of third-party evaluation constitutes a psychological foundation for indirect reciprocity (Nowak \& Sigmund, 1998): an observer who sees an individual (actor) helping another individual (recipient) may positively evaluate the actor and, thus, be more likely to help the actor in future interactions. In the same manner, observers may negatively evaluate anti-social individuals, and be less likely to help them in the future (Fehr, 2004; Nowak, 2006; Nowak \& Sigmund, 1998). Furthermore, language, a tool that transfers information beyond the here and now, plays one of crucial roles in sustaining humans' unique large-scale cooperative societies (Nowak \& Sigmund, 2005; Melis \& Semmann, 2010), because sharing reputations with others who have not directly experienced relevant events leads to a broader spread of positive or negative evaluations (Nowak \& Sigmund, 2005; Ohtsuki \& Iwasa, 2004).

The empirical findings and theoretical frameworks summarized above can help develop optimal strategies for individuals seeking to build positive reputations. Actors should focus on gaining a good reputation at minimal cost, which would enhance their chances of being helped in the future. This leads to a plausible optimal strategy for them: behaving cooperatively only in situations where reputation can be improved; namely, in the presence of observers (Engelmann \& Fischbacher, 2009; Izuma, Matsumoto, Camerer, \& Adolphs, 2011).

Meanwhile, observers should favor minimizing the gap between the estimation of an actor's helpfulness and the actor's authentic helpfulness. The reputation an observer holds about an individual, constructed from their own observations or through second- 
hand information, serves as material for choosing individuals to cooperate with (Nowak \& Sigmund, 2005). Thus, it would be more efficient for the observer to have a counterstrategy for detecting actors' intrinsic altruistic tendencies. As an actor's strategy of only cooperating in the presence of observers enlarges the gap between their estimated and actual altruism, it would be beneficial for observers to be epistemically vigilant concerning the contextual backgrounds of actors' behaviors, retaining some skepticism that the actors will perform identically in the absence of observers (Sperber et al., 2010). For example, when an actor's helpful behavior appears to be based on deliberate reputational management, observers no longer evaluate the behavior as they did previously.

We consequently hypothesized that creating a negative social evaluation upon discovering actors' calculating or strategic behaviors is a means of expressing such a counterstrategy. The detection and negative evaluation of norm violations may emerge early in development, as shown by a finding that six-month-old infants prefer actors who help others to those who hinder others (Hamlin, Wynn, \& Bloom, 2007). Meanwhile, five- to six-year-old children have been found to engage in indirect reciprocity in their natural daily interactions (Kato-Shimizu, Onishi, Kanazawa, \& Hinobayashi, 2013). These studies suggest that both children and infants socially evaluate them based on observations from the third-person perspective. Furthermore, reputational concerns were also found in preschoolers (Engelmann, Herrmann, \& Tomasello, 2012, 2018; Fu, Heyman, Qian, Guo, \& Lee, 2016). We could assume that preschoolers have some sensitivity to reputational management, based on these empirical facts.

The next question should be whether, and how, children attribute different reputational strategies to actors when they observe them from different observational contexts (Fu et al., 2016; Shaw \& Olson, 2015). Consequently, the current study developed an experimental setup featuring different observation contexts so that actors' apparent knowledge of the presence of observers could be controlled; in one context, an actor behaved while knowing that he/she was visible to the participant, whereas in another context, he/she behaved while believing he/she could not be seen, despite a participant watching the entire scene secretly. We used this format to present two forms of helper and measured the participants' relative preference.

As a first step, we investigated the effect of helping frequency, which matters in making social judgements (Nowak, 2006; Nowak \& Sigmund, 2005), in evaluating an agent with reputational management, and then two helpers were presented: one who behaved cooperatively in a calculating manner and another who neither engaged in such strategic behavior nor helped. To further test the effect of the actor's knowledge state of being observed induced by social context, the factor of helping frequency was eliminated. Two helpers were presented again, behaving cooperatively in a different social context. 


\section{Experiment 1: Test With Child Participants}

\section{MeTHOD}

We prepared two conditions: Public-only Helper vs. Unconditional Non-Helper (Condition 1) and Public-only Helper vs. Private-only Helper (Condition 2). Each participant participated in one of these two conditions.

\section{Participants}

Twenty-eight children recruited from the database of Kyoto University participated in Condition 1: 16 four-year-olds (seven girls; age $M=47.75$ months, $S D=0.83$, range $=47-50$ months) and 12 five-yearolds (six girls; age $M=60.58$ months, $S D=0.76$, range $=59-62$ months). Seven additional children were tested but were excluded from the sample due to experimental error $(n=2)$, fussiness during the session $(n$ $=1)$, or lack of response in the choice phase $(n=4$; explained in the later part).

Another twenty-eight children participated recruited from the database of Kyushu University in Condition 2: 15 four-year-olds (nine girls; age $M=48.33$ months, $S D=0.87$, range $=47-50$ months) and 13 five-year-olds (six girls; age $M=60.08$ months, $S D=1.33$, range $=57-62$ months). Participants who left the experimental booth before the end of the puppet event were classified as "fussiness during the session" and those who did not exhibit any reaction in response to the question by the experimenter were classified as "lack of response in the choice phase." Sixteen additional children were tested, but were excluded from the sample due to experimental error $(n=3)$, fussiness during the session $(n=8)$, or lack of response in the choice phase $(n=5)$.

\section{Ethics Statements}

The procedures were approved by the local ethical committee in the Unit for Advanced Studies of the Human Mind, Kyoto University and the Ethics Committee of the Faculty of Human-Environment Studies at Kyushu University. All child participants were recruited from a database of children whose parents had volunteered to participate in children's studies at Kyoto University or Kyushu University, respectively. Written informed consent was obtained from the children's caregivers before the experiment started.

\section{Common Materials and Methods}

\section{Apparatus}

Puppets. Eight puppets were prepared (Fig. 1). Each puppet represented an animal that could be recognized by the children (dog, mouse, pig, cat, rabbit, frog, bear, and hippopotamus). Three pseudorandomly selected puppets were used for each experiment session. To prevent prior exposure or original
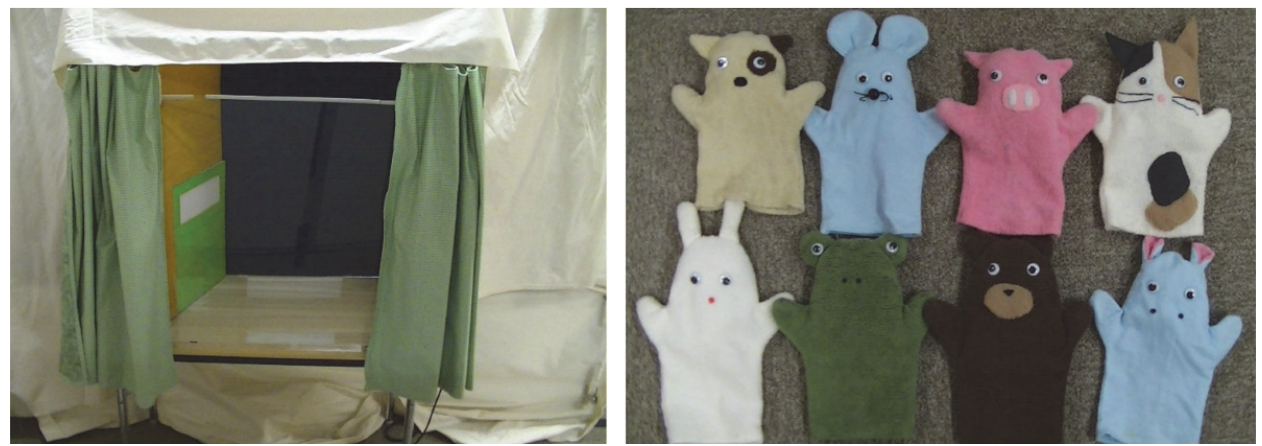

Fig. 1. The puppet stage and the puppets used in the current study 
preference for the puppets from affecting the target preference, they were hand-made by the first author, and any puppets representing animals that were liked or disliked by the participant (reported by the child's caregiver) were not used with that particular child. The frequency of usage and the role of each puppet were counterbalanced between participants as equally as possible.

Puppet stage. A puppet stage made of wooden boards (L $40 \mathrm{~cm} \times \mathrm{W} 91 \mathrm{~cm} \times \mathrm{H} 91 \mathrm{~cm})$ was placed on a table in one corner of the booth. On the stage, a door $(\mathrm{H} 36 \mathrm{~cm} \times \mathrm{W} 27 \mathrm{~cm})$ was located on the left side. A transparent acrylic plate was attached to the front of the stage. A pair of curtains was also placed at the front; these could be closed to occlude the stage. When the curtains were closed, they were held together with Velcro at three points. In the Private-context described below, the participant could peek at the stage through the narrow openings between the Velcro-fastened points (Fig. 1).

\section{Procedure}

Two experimenters conducted each session (E1 and E2). E1 manipulated the puppets and E2 observed the puppet events with the participants. Before the experiment, the participant and E2 played in a space adjacent to the experiment booth until the participant appeared to positively or spontaneously communicate with E2 and establish enough rapport that E2 could control the experiment situation. During this warm-up phase, E1 explained the experimental procedure to the caregiver and asked the latter not to interact with the child during the session. E1 was careful to have as little interaction as possible with the participant before the experiment, because the puppet interactions included E1's verbal dialogue. E1 then hid behind the puppet stage as E2 and the participant entered the experiment booth.

\section{Event}

In the experiment booth, the participant observed the puppet event on the stage. To minimize the possibility of any task-specific confounds influencing the results, two different events were shown to each participant. Shortly after one event described below was over, the same event with another agent commenced.

In the Door event, a puppet (Recipient) was carrying an object. Another agent then appeared on the stage from the right. The Recipient attempted to open the door and failed twice. Another puppet (Publiconly/Private-only/Unconditional Non-Helper) was then asked to open the door and either willingly helped or refused to help, depending on the situation. In the former situation, another puppet opened the door and the Recipient exited the stage through the door opened by another puppet. In the latter scenario, another puppet refused his offer by turning his back on him and a Recipient put an object on the floor and opened the door by himself. He then picked it up and exited the stage. Soon after his disappearance, another puppet exited the stage via the right side in both situations.

In the Box event, a puppet (Recipient) was dragging a box with a seemingly heavy load inside. Another agent then appeared on the stage from the right side. Another puppet (Public-only/Privateonly/Unconditional Non-Helper) was asked to carry the box and either willingly helped or refused to help, depending on the situation. In the former situation, they carried the box and exited the stage together. Soon after, one other agent (Public-only/Private-only/Unconditional Non-Helper) came back to the stage and left via the right side. In the latter case, another puppet refused his offer by turning his back on him and a recipient continued dragging the box alone and exited the stage via the left side while doing so. Soon after his disappearance, another agent exited the stage via the right side.

\section{Context}

Two different contexts were prepared: Public-context and Private-context. Before the start of the puppet event, the stage was obscured by curtains so that the observing contexts could be controlled, as explained below.

Public-context. E2 suggested that the child watch what was happening behind the curtain. E2 asked the puppets behind the curtains "Are you there? Can I open the curtain?", and soon after E2 opened the curtains, the above events commenced. The puppets on the stage show communicative behaviors toward E2 and the participant, such as by waving their heads to express goodbye when exiting the stage.

Private-context. E2 suggested that the child watch what was happening through the narrow openings in the curtains so that the puppets would not perceive the child. E2 spoke in whispers throughout this context. Contrary to the Public-context, the puppets showed no communicative behaviors toward the child 
and E2.

\section{Observation Phase}

Participants observed two helpers (Public-only Helper and Unconditional Non-Helper in Condition 1; Public-only Helper and Private-only Helper in Condition 2) perform two interactions in two different contexts respectively. Thus participants observed two successive interactions with a different helper in each context: the Public-only Helper helped a recipient only in the Public-context, the Private-only Helper helped a recipient only in the Private-context and the Unconditional Non-Helper refused to help across contexts.

The events (Door or Box events), the order of the contexts (Public-context first or Private-context first), and the order of appearance of the two types of helper were counterbalanced across participants. The appearance order of the helpers in each context was consistent for each participant.

\section{Choice Phase}

After the four interactions described above were completed, E1 placed two helpers side-by-side on the stage, with the curtains closed. Subsequently, E2 opened the curtains and asked "which do you prefer?" To exclude any behavioral cueing, the two puppets were fixed onto a bottle and the presentation position (left or right) was counterbalanced across participants. E2 remained silent until the participant made a response or 20 seconds had passed. Participants failing to express any choice within 20 seconds were excluded from the sample (classified as lack of response in the choice phase).

\section{RESULTS}

As coding the participant's choice, the puppet chosen in the choice phase, either verbally by name, or gesturally by pointing, was taken as the preferred one, and when participants verbally reported that they had no specific preference; for example "I like both", they were classified as no clear preference and excluded from further analyses (Condition1: five participants; Condition 2: one participant). The participants with lack of response did not show any response during choice phase were also excluded as mentioned in Participant section. The first author coded the responses of all participants, while another coder, blind to the experiment aim and the participants' choices, coded the videos for a random $25 \%$ of the sample. The inter-observer agreement was $100 \%$.

Over half (65.2\%) of the participants preferred a Public-only Helper more than an Unconditional Non-Helper in Condition 1 (Table 1). This result could be equivalent to a

Table 1. The number of participants (children) who preferred each agent in Experiment 1

\begin{tabular}{lcc}
\hline Condition & $\begin{array}{c}\text { Preferred } \\
\text { Public-only Helper }\end{array}$ & $\begin{array}{c}\text { Preferred } \\
\text { counter agent }\end{array}$ \\
\hline $\begin{array}{l}\text { Condition 1 } \\
\text { Public-only Helper } \\
\text { vs. }\end{array}$ & 15 & 8 \\
Unconditional Non-Helper & $(65.2 \%)$ & $(34.8 \%)$ \\
Condition 2 & & \\
Public-only Helper \\
$\quad$ vs.
\end{tabular}


"baseline" preference for the target agent; the latter agent was just a spectator and is not considered to contribute any effects of cooperative behavior or control other potential factors, such as exposure time, in forming social preference. In contrast, the preference for Public-only Helper in Condition 2 dropped to 33.3\% (Table 1); thus, to analyze effects of the presentation of the Private-only Helper on the preference for the Publiconly Helper, a chi-square test was applied across conditions.

Results indicated that the preference ratio for the agents between those two conditions were significantly different $\left(\chi_{(1, N=50)}^{2}=3.86, p<.05\right)$. The residual analyses indicated a preference among the children for the counter agent of the Public-only Helper in Condition 2 over the counter agent in Condition 1 (residual $=2.25, p<.05$ ).

The participants were asked to choose one of two agents so that we could directly analyze the difference between the numbers of children who showed a clear preference for the agents using binomial tests. However, we failed to detect differences in the children's preferences toward any particular agent in both conditions (two-tailed, $p s>$ $.10)$.

\section{DisCUSSION}

The majority of participants behaved differentially depending on the context. They were attempting to initiate social communication with puppets (waving their hands or talking to puppet) in Public-context. On the contrary, they inhibited communicative behavior and were whispering so that they would not be found by the puppets in Privatecontext. Therefore, it would be reasonable to interpret that our manipulation on the observation contexts worked appropriately.

The preference for a Public-only Helper in Condition 1 was relatively high compared to Condition 2. This finding is consistent with previous studies showing that children inherently prefer cooperative agents (Hamlin et al., 2007) and those who help with high frequency (Koenig \& Harris, 2005). The preference for a Public-only Helper was impaired more by the presence of a Private-only Helper than an Unconditional NonHelper. This result could be attributed merely to the presence of another agent who helped more frequently than the Unconditional Non-Helper; the Private-only Helper helped more frequently than the Unconditional Non-Helper (50\% and $0 \%$, respectively). However, it can also be interpreted that the children's tendency to prefer the Public-only Helper was weakened in Condition 2 compared to Condition 1. The decrement in the preference for a Public-only Helper compared to a Private-only Helper from "baseline" might be attributed to the fact that children preferred the Private-only Helper.

The direct comparison between two agents within each condition via binomial tests did not reach significance. It is possible that children had sensitivity to social context and helping frequency, and that the latter factor might have impaired the positive effect of high helping frequency to the level where any difference was detected. Indeed, marginally more participants appeared to have difficulty with social evaluation in Condition 1 than in Condition 2; in Condition 1, two factors (social context and helping 
frequency) played a competing (contradicting) role, unlike the latter condition which controlled for helping frequency. Anecdotal evidence also supports sensitivity among children not only for helping frequency, but also for social context. In Condition 1, three children who preferred the Public-only Helper reported doing so because of his helping; the one participant who preferred the Unconditional Non-Helper reported that he preferred him because he did not help. We must remain cautious in drawing conclusions as the results of the binomial test and this anecdotal evidence is not direct evidence for the presence of such sensitivity. It would suggest, however, that the context with two contradicting evaluation criteria imposed difficulty in making social judgement; thus, children less preferred the Public-only Helper, resulting from the effect derived from both factors.

\title{
Experiment 2: Test With Adult Participants
}

Experiment 2 examined how adults socially evaluated agents with or without reputational management. In addition to the conditions applied for children, another condition with new agent, Unconditional Helper who helped regardless of being seen, was also tested; the Private-only Helper vs. Unconditional Helper condition (Condition 3 ), to test the effect of the helping frequency outside of the context of calculating strategy.

\section{METHOD}

\begin{abstract}
Participants
We tested forty adults participants (16 females; age $M=23.55$ years, $S D=3.23$, range $=18-36$ years).

Apparatus and Procedure

We prepared picture books representing identical sequences to Experiment 1, using stills from videorecordings of the puppet events (Fig. 2). The fully open image was overlaid on all scenes in the Publiccontext, and the slightly open image was overlaid on all scenes in the Private-context. To emphasize the difference between the contexts, the backgrounds of the sheets were colored differently: yellow for Publiccontext and grey for Private-context. The participants were instructed to imagine that the puppet events were occurring in front of them and that they were observing them either directly or by peeking through the narrow opening between the closed curtains.

Participants were tested under Conditions 1-3. We prepared different versions of picture-book sets for each condition, which counterbalanced potential artefacts, such as event, appearance order, context order and puppets' roles, and randomly assigned each participant to one of the three sets. After observing each condition, participants noted their preference on the corresponding sheets.
\end{abstract}

\section{Results}

As in Experiment 1, the relative preference for Public-only Helper comparting with 


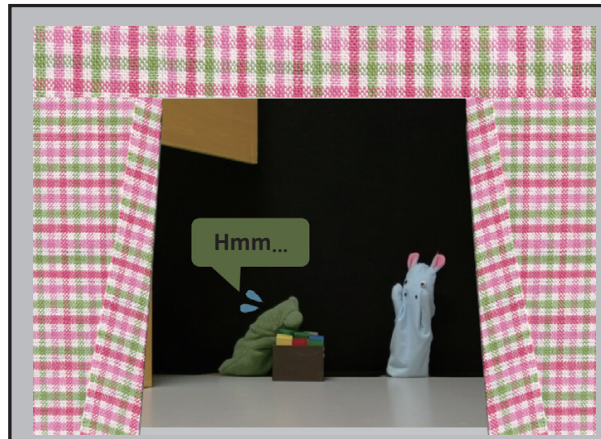

The frog is dragging a box. Then, a hippopotamus appears. He seems to not be aware of your presence.

(A)

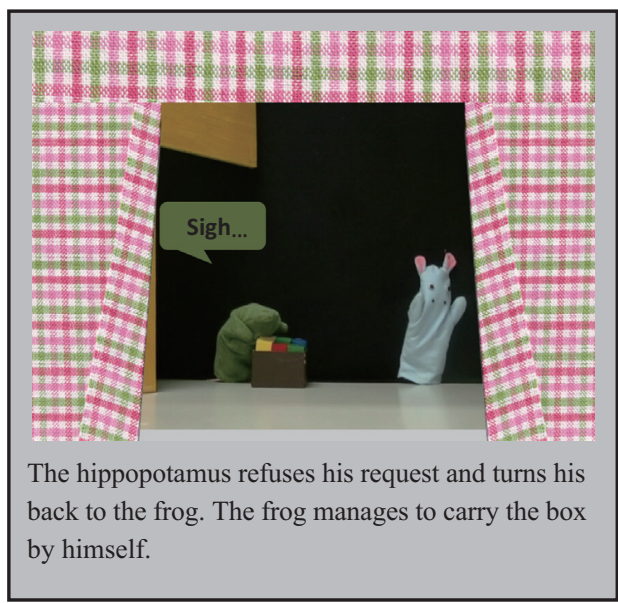

(C)

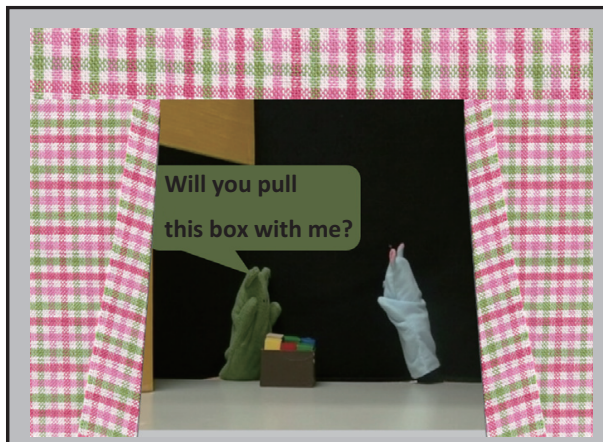

The frog asks the hippopotamus to pull the box together.

(B)

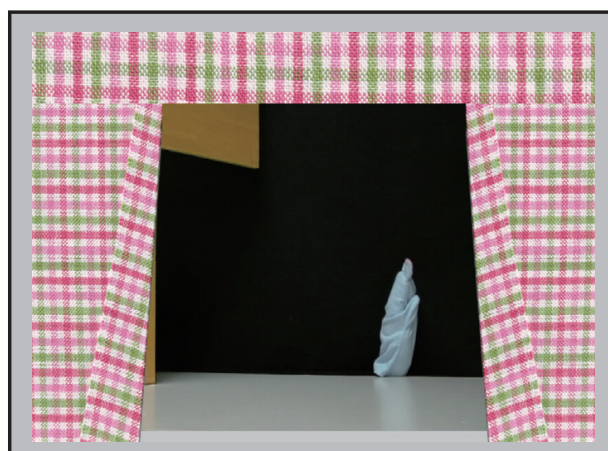

The hippopotamus exits. He remains unaware of your presence.

counter agents were analyzed. The percentage who chose each agents was not significantly different between conditions $\left(\chi_{(1, N=80)}^{2}=0.65, p>.10\right)$, suggesting that adults had the same level of avoidance of the Public-only Helper, irrespective of compared agents (Table 2).

Furthermore, in contrast to the results of children, binomial tests revealed that participants chose Unconditional Non-Helper over Public-only Helper (29/40, twotailed, $p<.01$ ) in Condition 1 and Private-only Helper significantly more over Publiconly Helper (33/40, two-tailed, $p<.001$ ) in Condition 2. Similarly, more participants chose Unconditional Helper over Private-only Helper (33/40, two-tailed, $p<.001)$ in Condition 3. 


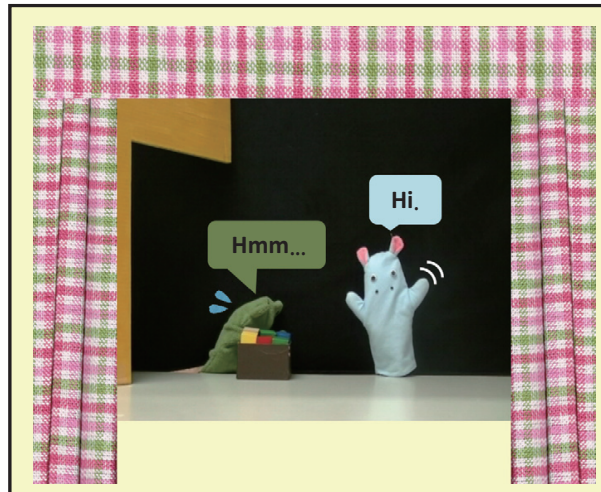

The frog is dragging a box. Then, a hippopotamus appears, becomes aware of your presence, and greets you.

(A)

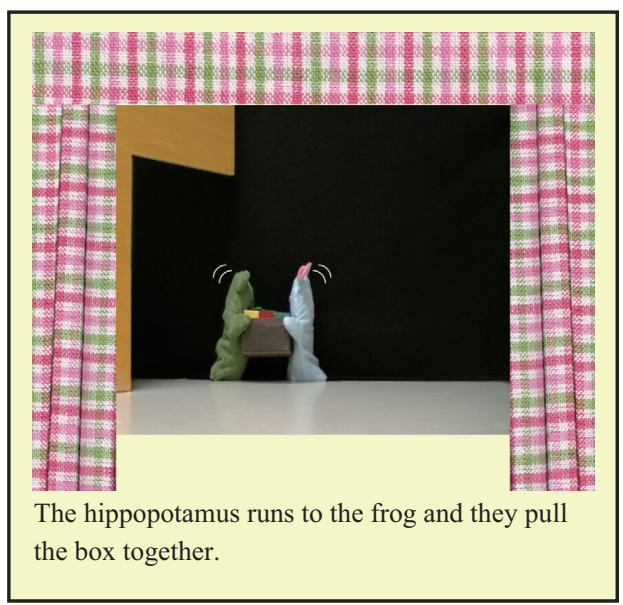

(C)

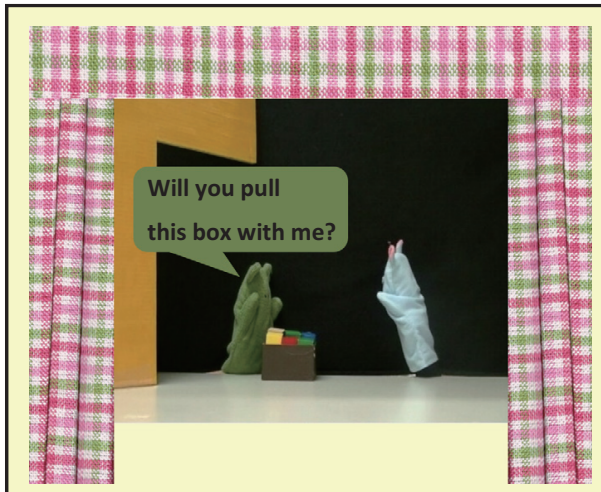

The frog asks the hippopotamus to pull the box together.
(B)

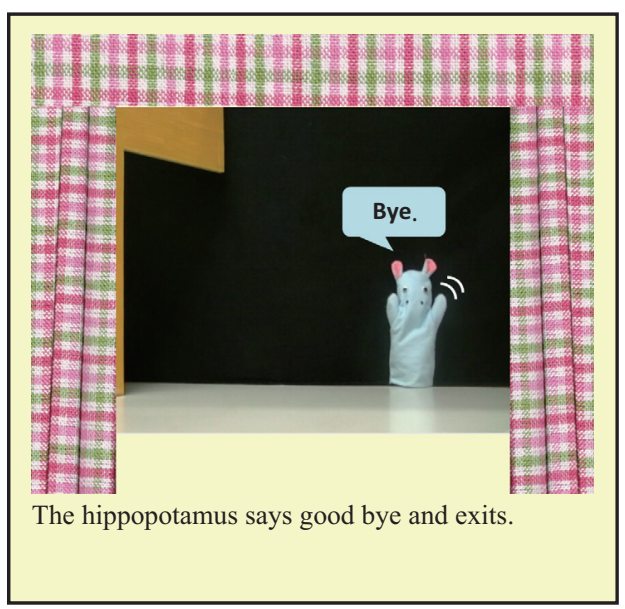

(D)

Fig. 2. Example of picture books used in the adult experiment. The former set of illustrations is from the Private-contexts. The latter set is from the Public-contexts.

\section{Discussion}

Adult participants negatively evaluated the Public-only Helper compared to the Private-only Helper; adults seemed to give more weight to the Private-context in evaluating others. Furthermore, within the context of a calculating strategy, participants showed a preference that did not depend on high helping frequency: they preferred the Unconditional Non-Helper to the Public-only Helper. In contrast, outside of the context of a calculating strategy, high helping frequency did matter in evaluating others: they preferred the Unconditional Helper to the Private-only Helper. Their disapproval of calculating behavior surpasses the approval gained through frequent observed helping. 
Table 2. The number of participants (adults) who preferred each agent in Experiment 2

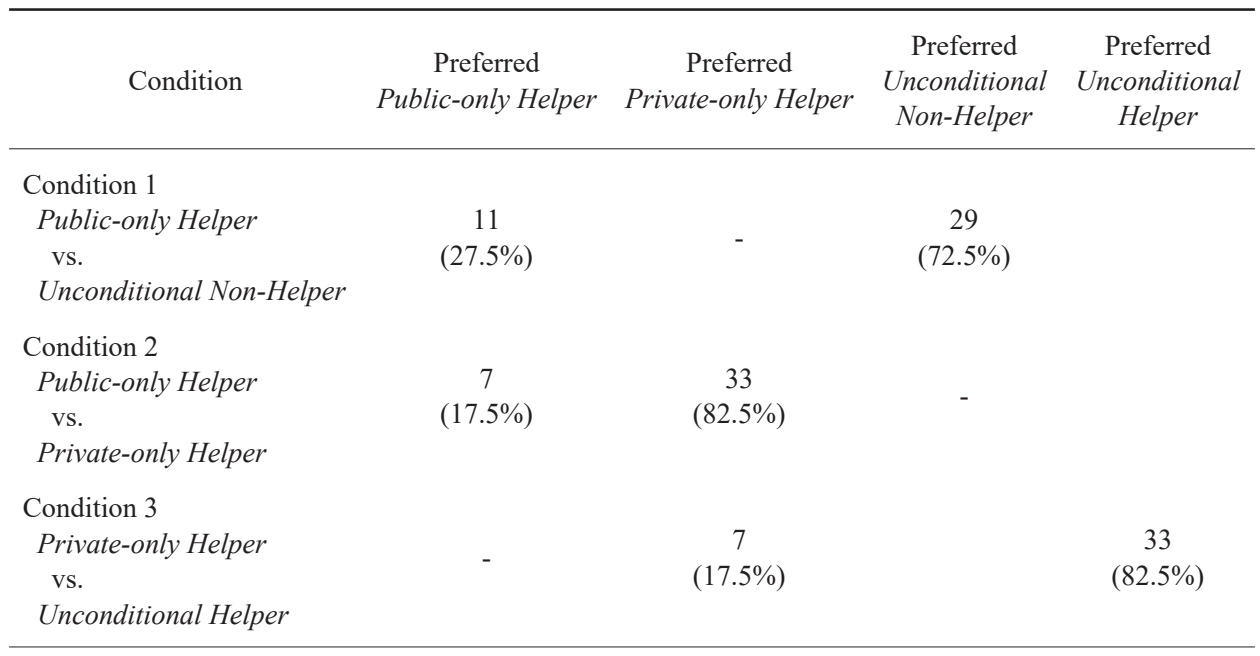

This argues that, in adults, the tendency to avoid a calculating strategy might have a stronger influence on the expression of preference than helping frequency.

\section{General Discussion}

In the current study, four- to five-year-olds showed neither preference nor avoidance for a Public-only Helper when investigating the effect of helping frequency, controlling for helping frequency, and investigating the effect of social context. However, our results suggest that children less preferred a Public-only Helper when a Private-only Helper was presented than when an Unconditional Non-Helper, a baseline agent eliminating the effect of cooperative behavior, was presented. The experiment with adult participants showed that adults robustly avoided Public-only Helpers; unlike children, they showed avoidance for Public-only Helpers beyond the advantage of high helping frequency. These results suggest that children may have a social evaluation for reputational management similar to that of adults, in that they did not prefer Public-only Helpers; however, this result might be dependent on the advantage of high helping frequency and not as robust and strong as in adults. However, the current study excluded relatively high number of children $(29 / 79)$ from the analyses. Thus, we have to be cautious that this effect can be generalized to this age group as a whole, since the analyses were made from participants with keeping their focus on the puppet story until the event was over and with exhibiting specific preference.

A calculating strategy would be advantageous if all private interactions were really kept private (consequently having no influence on reputation). However, as schematized in the current study, some private interactions can be detected by others. The cognitive bias for the observer to emphasize information gained from private interactions functions 
as a counterstrategy to calculating strategies, thereby enabling stable cooperation (Trivers, 1971; Ohtsuki \& Iwasa, 2006). A recent theoretical study demonstrated that cooperation eventually becomes optimal, irrespective of the probability of being observed (provided the probability is not completely zero; Ohtsuki, Iwasa, \& Nowak, 2015). Interestingly, their model assumes that individuals have different assessment rules for public and private interactions. The current results provide behavioral evidence that, in the context of third-party observation, four- to five-year-old observers apply different social evaluations in contexts where the observed interaction is supposed to be public or private.

From a utilitarian point of view (Yamagishi, Li, Takagishi, Matsumoto, \& Kiyonari, 2014), it would be reasonable for participants to prefer the Public-only Helper under the comparison of agents with equal helping frequency, because the helper's helpfulness was easily controllable and predictable by their presence; however, our participants -slightly in children and robustly in adults- showed a preference for the helper who helped only in private. Consequently, the question arises: how does interaction in the Private-context, with such uncertainty, contribute more effectively to estimation of the helpfulness of future interactions?

When an individual has an opportunity to behave altruistically, they generally do not know beforehand whether the interaction will be public or private. Thus, observers might interpret cooperation in the Public-context as controlled and driven by explicit social pressure, not a complete expression of the individual's actual altruistic tendency; in contrast, they might interpret cooperation in the Private-context as reflecting the actor's true altruistic tendencies. Considering the relatively few chances of observing behavior in a private, this tendency might reflect a more general psychological bias towards attributing greater weight to rare events or phenomena (McKinnon \& Servedio, 2013).

Another important finding here is that the level by which helping frequency influences social preference differed between children and adults. Adults' avoidance of the Public-only Helper was too strong to be influenced by the advantage of high helping frequency; however, the young children's evaluation seemed not to be dependent on the effect of high helping frequency. Further studies should clarify whether the underlying psychological mechanism of these preferences is based on utilitarian (Yamagishi et al., 2014) or sympathetic tendencies (i.e., young children primarily feeling that others in need should be helped; Hepach, Vaish, \& Tomasello, 2012). Our findings suggest that sensitivity to helping frequency, including reputational management, might vary through development.

Our methodology incorporating peeking behavior opened up a new path to explore false belief (Baron-Cohen, Leslie, \& Frith, 1985; Wimmer \& Perner, 1983) rooted in the concept of Theory of Mind (Premack \& Woodruff, 1978; cf. Wellman, 1991), enabling us to create an experimental situation in which the observer and actor had different knowledge states. To summarize the logical requirements of the current study, the participant had to understand "I know that they (puppets) behaved with/without knowing that I know they noticed my presence." Most of the tasks exploring the second-order false belief in children (Miller, 2009; Perner \& Wimmer, 1985; Sullivan, Zaitchik, \& 
Tager-Flusberg, 1994) required significant cognitive load, that is, children had to remember several agents and follow a complex story. Thus, the results of those tasks might be attributed to aspects of cognitive development other than false-belief understanding. In fact, several studies that have used simplified test settings showed that children passed first-order belief tasks earlier than previously proposed (Norimatsu, Blin, Hashiya, Sorsana, \& Kobayashi, 2014; Onishi \& Baillargeon, 2005; Southgate, Senju, \& Csibra, 2007). The paradigm used in the current study reduced cognitive load by allowing observation of the event from the first-person perspective. It must be noted that although our study did not require reporting of a verbal understanding of false belief, the current methodology surely contributed to the onset of implicit processing of false belief, and thus, the nested structures of mind. In closing, the current results provide evidence that four- to five-year-old children might already possess a tendency to less prefer reputation management, although it is not as robust or salient as in adults. Whether, when, and what interactions between genetic and socio-cultural backgrounds influence this biased social evaluation in development is still an open question. Additionally, the transition from children's mindsets to the tendency shown in adults of avoiding calculating agents is another important topic for future studies. This bi-directional exploration is certain to contribute to our understanding of the ontogeny of social evaluations, a psychological foundation of human society.

\section{COMPETING INTERESTS}

The authors declare no competing financial interests.

\section{AUTHOR'S CONTRIBUTIONS}

R.K. and K.H. conceived the experiments; R.K. performed the experiments and R.K. analyzed the data. R.K., K.H., S.I., and K.F. contributed in interpreting the results. R.K. and K.H. wrote the paper. All authors gave final approval for publication.

\section{REFERENCES}

Baron-Cohen, S., Leslie, A. M., \& Frith, U. (1985). Does the autistic child have a "theory of mind"? Cognition, 21, 37-46.

Bshary, R., \& Grutter, A. S. (2006). Image scoring and cooperation in a cleaner fish mutualism. Nature, 441, 975-978.

de Waal, F. B. (1989). Food sharing and reciprocal obligations among chimpanzees. Journal of Human Evolution, 18, 433-459.

Engelmann, D., \& Fischbacher, U. (2009). Indirect reciprocity and strategic reputation building in an experimental helping game. Games and Economic Behavior, 67, 399-407.

Engelmann, J. M., Herrmann, E., \& Tomasello, M. (2012). Five-year olds, but not chimpanzees, attempt to manage their reputations. PLOS ONE, 7, e48433. 
Engelmann, J. M., Herrmann, E., \& Tomasello, M. (2018). Concern for group reputation increases prosociality in young children. Psychological Science, 29, 181-190.

Fehr, E. (2004). Don't lose your reputation. Nature, 432, 449-450.

Fu, G., Heyman, G. D., Qian, M., Guo, T., \& Lee, K. (2016). Young children with a positive reputation to maintain are less likely to cheat. Developmental Science, 19, 275-283.

Fujisawa, K. K., Kutsukake, N., \& Hasegawa, T. (2008). Reciprocity of prosocial behavior in Japanese preschool children. International Journal of Behavioral Development, 32, 89-97.

Hamlin, J. K., Wynn, K., \& Bloom, P. (2007). Social evaluation by preverbal infants. Nature, 450, 557-559.

Hepach, R., Vaish, A., \& Tomasello, M. (2012). Young children are intrinsically motivated to see others helped. Psychological Science, 23, 967-972.

Izuma, K., Matsumoto, K., Camerer, C. F., \& Adolphs, R. (2011). Insensitivity to social reputation in autism. Proceedings of the National Academy of Sciences of the United States of America, 108, 17302-17307.

Kato-Shimizu, M., Onishi, K., Kanazawa, T., \& Hinobayashi, T. (2013). Preschool children's behavioral tendency toward social indirect reciprocity. PLOS ONE, 8, e70915.

Koenig, M. A., \& Harris, P. L. (2005). Preschoolers mistrust ignorant and inaccurate speakers. Child Development, 76, 1261-1277.

Liszkowski, U., Carpenter, M., Striano, T., \& Tomasello, M. (2006). 12- and 18-month-olds point to provide information for others. Journal of Cognition and Development, 7, 173-187.

Liszkowski, U., Carpenter, M., \& Tomasello, M. (2008). Twelve-month-olds communicate helpfully and appropriately for knowledgeable and ignorant partners. Cognition, 108, 732-739.

McCabe, K., Houser, D., Ryan, L., Smith, V., \& Trouard, T. (2001). A functional imaging study of cooperation in two-person reciprocal exchange. Proceedings of the National Academy of Sciences of the United States of America, 98, 11832-11835.

McKinnon, J. S., \& Servedio, M. R. (2013). Novelty makes the heart grow fonder. Nature, 503, 44-45.

Melis, A. P., \& Semmann, D. (2010). How is human cooperation different? Philosophical Transactions of the Royal Society B, 365, 2663-2674.

Milinski, M. (1987). TIT FOR TAT in sticklebacks and the evolution of cooperation. Nature, 325, $433-435$.

Miller, S. A. (2009). Children's understanding of second-order mental states. Psychological Bulletin, 135, $749-773$.

Norimatsu, H., Blin, R., Hashiya, K., Sorsana, C., \& Kobayashi, H. (2014). Understanding of others' knowledge in French and Japanese children: A comparative study with a disambiguation task on 16-38-month-olds. Infant Behavior and Development, 37, 632-643.

Nowak, M. A. (2006). Five rules for the evolution of cooperation. Science, 314, 1560-1563.

Nowak, M. A., \& Sigmund, K. (1998). Evolution of indirect reciprocity by image scoring. Nature, 393, 573-577.

Nowak, M. A., \& Sigmund, K. (2005). Evolution of indirect reciprocity. Nature, 437, 1291-1298.

Ohtsuki, H., \& Iwasa, Y. (2004). How should we define goodness? - reputation dynamics in indirect reciprocity. Journal of Theoretical Biology, 231, 107-120.

Ohtsuki, H., \& Iwasa, Y. (2006). The leading eight: Social norms that can maintain cooperation by indirect reciprocity. Journal of Theoretical Biology, 239, 435-444.

Ohtsuki, H., Iwasa, Y., \& Nowak, M. A. (2015). Reputation effects in public and private interactions. PLOS Computational Biology, 11, e1004527.

Olson, K. R., \& Spelke, E. S. (2008). Foundations of cooperation in young children. Cognition, 108, $222-231$.

Onishi, K. H., \& Baillargeon, R. (2005). Do 15-month-old infants understand false beliefs? Science, 308, $255-258$.

Perner, J., \& Wimmer, H. (1985). "John thinks that Mary thinks that..." attribution of second-order beliefs by 5- to 10-year-old children. Journal of Experimental Child Psychology, 39, 437-471.

Premack, D., \& Woodruff, G. (1978). Does the chimpanzee have a theory of mind? Behavioral and Brain Sciences, 1, 515-526.

Shaw, A., \& Olson, K. (2015). Whose idea is it anyway? The importance of reputation in 
acknowledgement. Developmental Science, 18, 502-509.

Southgate, V., Senju, A., \& Csibra, G. (2007). Action anticipation through attribution of false belief by 2year-olds. Psychological Science, 18, 587-592.

Sperber, D., Clément, F., Heintz, C., Mascaro, O., Mercier, H., Origgi, G., \& Wilson, D. (2010). Epistemic vigilance. Mind \& Language, 25, 359-393.

Sullivan, K., Zaitchik, D., \& Tager-Flusberg, H. (1994). Preschoolers can attribute second-order beliefs. Developmental Psychology, 30, 395-402.

Tomasello, M. (2009). Why we cooperate. Cambridge, MA: MIT Press.

Trivers, R. L. (1971). The evolution of reciprocal altruism. The Quarterly Review of Biology, 46, 35-57.

Wellman, H. M. (1991). From desires to beliefs: Acquisition of a theory of mind. In A. Whiten (Ed.), Natural theories of mind: Evolution, development and simulation of everyday mindreading (pp. 19-38). Oxford, United Kingdom: Basil Blackwell.

Wilkinson, G. S. (1984). Reciprocal food sharing in the vampire bat. Nature, 308, 181-184.

Wimmer, H., \& Perner, J. (1983). Beliefs about beliefs: Representation and constraining function of wrong beliefs in young children's understanding of deception. Cognition, 13, 103-128.

Yamagishi, T., Li, Y., Takagishi, H., Matsumoto, Y., \& Kiyonari, T. (2014). In search of Homo economicus. Psychological Science, 25, 1699-1711.

(Manuscript received 22 November, 2018; Revision accepted 22 August, 2019; Released online in J-STAGE as advance publication 3 April, 2020) 